\title{
Integrating Activity Based Costing and Process Simulation for Strategic Human Planning
}

\author{
Takayuki Kataoka ${ }^{1}$, Aritoshi Kimura ${ }^{1}$, Katsumi Morikawa ${ }^{2}$ and \\ Katsuhiko Takahashi ${ }^{2}$ \\ 1 Department of Information Systems Engineering, \\ Faculty of Engineering, Kinki University, \\ Takaya Umenobe 1, Higashi-Hiroshima, Hiroshima, 739-2116, JAPAN \\ \{kataoka, kimura\}@hiro.kindai.ac.jp \\ WWW home page: http://www.hiro.kindai.ac.jp \\ 2 Department of Artificial Complex and System Engineering, \\ Graduate School of Engineering, Hiroshima University, \\ 4-1 Kagamiyama 1-chome, Higashi Hiroshima, 739-0857, JAPAN
}

\begin{abstract}
This paper presents a method of integrating activity-based costing $(A B C)$ and process simulation in human planning. Our studies have already proposed a method of integrating $A B C$ and process simulation in business process reengineering (BPR) and showed a case study of a chemical plant. Some studies have also already showed some examples of various aspects in manufacturing systems. Although a large number of studies have been made on product-mix/machine loading or scheduling, little is known about human planning. In this paper, effective BPR methodologies to achieve dramatic improvements in business measures of workers' skills and costs based on $A B C$ are discussed. First, two important tools: process simulation method and $A B C$ analysis that can be customized by organizations for their own BPR are shown. As these tools have been separately used, a unified approach of process simulation and $\mathrm{ABC}$ analysis for process redesigns based on simulation. Secondly, a method of process simulation design is shown. It is repeated to consider working ratio and running time of resources. Thirdly, a method of $\mathrm{ABC}$ analysis that can be customized by organizations is shown. It automatically shows the data that has been gathered from many sources. By utilizing the data, the process simulation is implemented, and the result of simulation gives the data to $\mathrm{ABC}$ analysis. Lastly, this paper shows a case study in BPR and the effectiveness of our method.
\end{abstract}

\author{
Keywords \\ Activity-Based Costing (ABC), Human Planning, Process Simulation
}

Please use the following format when citing this chapter:

Kataoka, T., Kinura, A., Morikawa, K., Takahashi, K., 2007, in IFIP International Federation for Information Processing, Volume 246, Advances in Production Management Systems, eds. Olhager, J., Persson, F., (Boston: Springer), pp. 273-280. 


\section{Introduction}

Recently, there has been a great deal of interest in business process reengineering (BPR). Hammer and Champy [1] defined reengineering as "the fundamental rethinking and radical redesign of core business processes to achieve dramatic improvements in quality, cost, and cycle time". Earlier literature on BPR focused only on the principles of BPR and provided us with cases of successful BPR projects that address only the "what" and "why" questions [2]. However, there are few comprehensive and methodological approaches to conducting BPR projects to answer to the "how" question. Therefore, this paper focuses on development of comprehensive BPR methodologies. This paper first describes the process simulation method and the activity-based costing (ABC) analysis, which are likely considered as the most effective methods and tools in BPR methodologies. The former is effective to analyze whether the newly designed process can meet performance standards. "What if" questions related to process performance may be asked. The latter is a very useful tool when cost saving is the major goal of a BPR project. However, both are usually used separately in a BPR project. It can be seen clearly that rather than using these methods separately, it is better to integrate both methods in doing reengineering process. Therefore, this paper proposes a new methodology, ABPCSS (Activity-Based Process and Costing Simulation System), which explicitly unified both process simulation method and activity-based costing analysis. At last, this paper also gives a simple case study of strategic human planning about the use of the unified approach.

\section{BPR Methods and Tools: Process Simulation Method and Activity-Based Costing(ABC) Analysis}

There are several methods and tools for process modeling and analysis. Among them, the process simulation method and the $\mathrm{ABC}$ analysis are especially effective to the understanding of complex business processes.

\subsection{Process Simulation}

Strictly speaking, simulation is the use of a model as a basis for exploration and experimentation. Like all modeling approaches, simulation is used because it is cheaper, safer, quicker, and more secure than using the real system itself. The idea is that the model becomes a vehicle for asking "what-if" questions. That is, the simulation model is subject to known inputs and the effects of these inputs on the outputs are noted.

Whatever the simulation modeling approach adopted, a simulation model must be able to mimic the changes that occur, through time, in the real system. The majority of applications of dynamic simulation methods in management science seem to use discrete event models [3].

The simulated process is summarized into four steps:

1. Specify model. 
2. Build model.

3. Simulate model.

4. Use model.

Each step is conducted as follows:

Step 1: Specify model.

Describe the objectives of the system to be simulated, which is in discrete condition where the situation change as time goes. The following things are described:

- Entity: tangible component of the system.

- Class: grouping of similar objects that share similar features or attributes.

Moreover, to describe how entity changes as time goes, the following things are also described:

- State: one for a period of time that is occupied by entities.

- Event: a state at which the entity changes state.

- Activity: one that the entity is changing state and this takes time to happen.

- Process: chronological sequence of activities.

Step 2: Build model.

In order to build a simulation model, we must understand the logic of the system to be simulated in terms of the entities and their interactions. In order to do that, an activity cycle diagram (ACD), one of the description methods, was made. An ACD only has two symbols: active state and dead state, as follows:

- Active state: one whose time duration can be directly determined at the event which marks its start.

- Dead state: one whose duration cannot be so determined but can only be inferred by knowing how long the active states may last.

Step 3: Simulate model (by VIMS (Visual Interactive Simulation and Modeling System)).

The usual way in which a VIMS model is developed is to begin with a blank background screen and then to pace icons on the screen to represent the major components of the system. These icons are then linked together by drawing lines on the screen to form a type of network, which captures the logical interactions between the entities of the system.

Step 4: Use model.

The use of the model involves the making of runs and the interpretation and presentation of the outputs. When simulation results are used to draw inferences or to test hypotheses, statistical methods should be employed.

\subsection{Activity-based costing (ABC)}

$\mathrm{ABC}$ is a technique that measures the cost of activities. Costs that are generally treated as overhead are allocated to activities identified in the process model. ABC can provide insight into overhead.

There are 5 steps in an $\mathrm{ABC}$ analysis, as follows:

1. Analyze activities.

2. Gather costs.

3. Trace costs to activities. 
4. Establish output measures.

5. Analyze costs.

We describe each of these steps in detail here [4]:

Step 1: Analyze activities.

This step conducts process decomposition, call "bill of activities", in the ABC effort. Then, activities are classified as primary, secondary, required, or discretionary. A primary activity is one that directly supports the organization's mission. Secondary activities support primary activities. Activities that the organization must be performed are required, while discretionary activities are ones that are truly optional.

Step 2: Gather costs.

The next step gathers costs for each activity. Collecting costs could be the most difficult aspect of conducting $\mathrm{ABC}$ because of lack of historical data for existing activities. The overhead cost of producing products or delivering service often represents a larger percentage of total costs than material, labor, or machinery.

Step 3: Trace costs to activities.

Cost is traced to an activity based on the charges for each activity. Resources used by activities, and hence their costs, are traced to activities. The total input cost of each secondary activity is allocated to the primary activities it supports. Total input cost for each activity can be calculated at this stage.

Step 4: Establish output measures.

Each activity may have more than one output. One primary output must be identified and it should be quantifiable. The unit cost of an activity is calculated by dividing the total input cost of the activity by its primary output volume.

Step 5: Analyze costs.

Cost analysts can use cost driver analysis to identify activities or factors that influence the cost and performance of other activities. The cause for high cost should be treated at its source. Cost data can be analyzed based on cost elements (i.e., labor, material, service, and supplier) in order to compare alternative designs to the baseline. Non-value-added activities are targets for elimination or improvement.

\section{A Unified Approach: Integrating Process Simulation and Activity-Based Costing (ABC)}

In general, it is better to use some methods and tools as one unit than use them separately. Even though there have been a lot of BPR methods and tools developed, research concerning a unified approach is scarce [5]. Therefore, we try to develop a new system methodology for BPR: an Activity-Based Process and Costing Simulation System (ABPCSS), which unified the largely used BPR methodologies: the process simulation method and the activity-based costing analysis. We explain this below. The primary components of ABPCSS are:

- Database system: This is a set of related files in the ABPCSS. Resources, activities, and cost objects are stored in the files.

- Activity-based process simulator: This uses the simulation model to forecast the performance of reengineered processes. 
- Activity-based costing simulator: This calculates the detailed cost of resources consumed by activities and the cost of activities consumed by the cost objects within the existing and reengineered processes.

- Graphical user interface: This allows user to communicate with any component of the ABPCSS.

Figure 1 shows data flow among each system component.

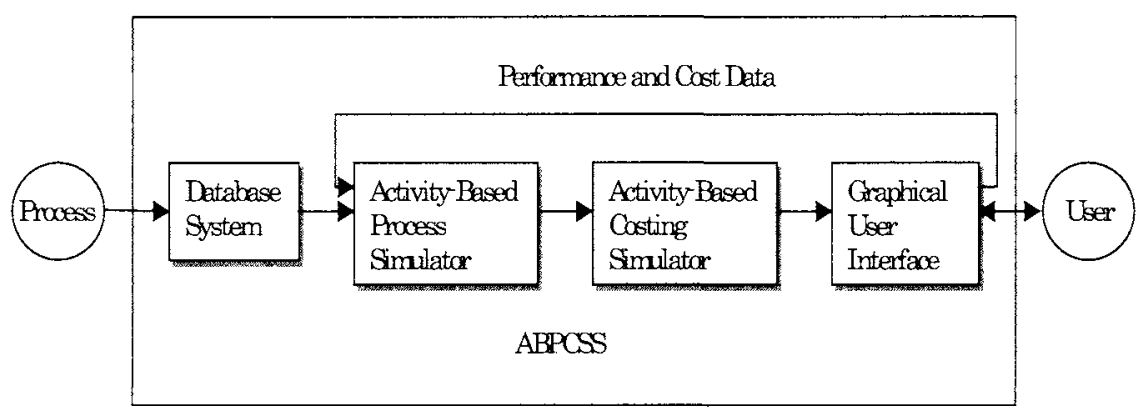

Fig. 1. A framework of activity-based process and costing simulation system (ABPCSS)

The process data and the bill of activities can be fed into the activity-based process simulator to analyze the performance of an existing process, and then performance and cost data can be fed into the activity-based costing simulator in order to analyze the cost of existing process. Then, the performance and cost data can be used to compare the reengineered process with the existing process. Design of the new process may be revised, and then re-evaluated with the ABPCSS until a finalized process is determined.

\section{A Case Study: Small-Scale Cellular Manufacturing Operation}

In order to illustrate the above procedures, we will take up the reengineering problem of the small-scale cellular manufacturing operation, which is made by using Arena, one of VIMS. This is conducted by a number of workers, which execute various jobs involving collaborative operations. Therefore, how to allocate single and collaborative operations to appropriate number of workers becomes an important topic in the equipment factory.

\subsection{Modeling for Simulation}

First, a simulation model concerning operations for such small cellular manufacturing workers is expressed, where the objective is to find improvement points. Preconditions for small cellular manufacturing operation discussed here, are as follows:

- The kinds of materials: 3

Materials 1: average working time: 50 minutes, transportation time: 10 minutes 
Materials 2: average working time: 75 minutes, transportation time: 12 minutes Materials 3: average working time: 40 minutes, transportation time: 8 minutes

- The number of cells: 5

- The number of processes in each cell: 4

- Each process needs the specified skills

- About processes in each cell:

1st process: the skill must be more than level 6, 2nd process: more than level 5 , 3th process: more than level 4, 4th process: more than level 6

- Organization: fixed 5 workers $(A, B, C, D, E)$

- Each worker's skill consists of 10 degrees:

Worker A: skill level 8: 28 dollars/hour, Worker B: skill level 7: 28 dollars/hour Worker C: skill level 7: 28 dollars/hour, Worker D: skill level 8: 28 dollars/hour Worker E: skill level 8: 28 dollars/hour

- Processing time per a day is 8 hours and a half (including break time: 30 minutes)

- Each worker is fixed in each cell

\subsection{Implementation and Analysis}

The average value of 10 working days is considered as simulation results. WIP (work-in-process) is started from zero. If it exists on the end of a working day, it is made on the next day.

Case A: 5 workers is fixed in each cell

The results of simulation are shown in Table 1 and 2. Based on these results, "Total Cost", "Idle Cost", and "Number Out" are focused to search for better results.

Table 1. An example of simulation results

\begin{tabular}{cccc}
\hline Total Cost & Busy Cost & Idle Cost & Number Out \\
\hline 35,519 & 22,473 & 13,047 & 140
\end{tabular}

Table 2. Instantaneous Utilization

\begin{tabular}{ccccc}
\hline Worker A & Worker B & Worker C & Worker D & Worker E \\
\hline 0.9699 & 0.5699 & 0.9824 & 0.9154 & 0.9773
\end{tabular}

\subsection{Optimization simulation}

Preconditions for optimization simulation discussed here, are as follows:

- Organization is classified in 4 groups

Group 1: Main Staff, G2: Main Staff2, G3: Sub Staff, G4: Sub Staff2

- Working place of each group

Main Staff : 2 processes in Cell 1 3, Main Staff2: 2 processes in Cell 4 or 5

Sub Staff : 2 processes in Cell $1 \sim 3$, Sub Staff2: 2 processes in Cell 4 or 5

Case B: how many temporary workers of 8 working hours per a day should be added as the optimal simulation? 


\section{- Optimized procedure}

Step 1: Maximum of "Number Out"

Step 2: Minimum of "Total Cost"

Step 3: Minimum of "Idle Cost"

(The maximum number of added workers is 12)

Step 1: Maximum of "Number Out".

These results are implemented by "Out Quest for Arena". Figure 2 shows the process of optimization. The optimal value is indicated at about 30 times. However the result of 204 products is lead by adding 4 temporary workers, it is not only one. Therefore, optimization of "Number Out" is added to the precondition, and the minimum of "Total Cost" and "Idle Cost" are similarly implemented by optimization simulation.

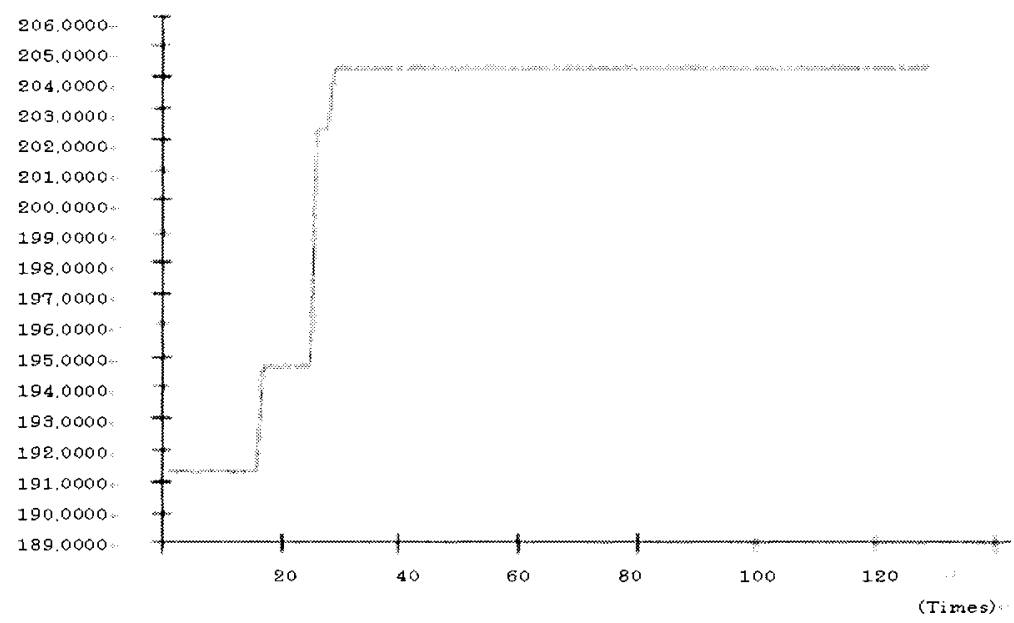

Fig. 2. Optimal solution for Step 1

Step 2: Minimum of "Total Cost"

The optimal value is indicated at about 30 times.

- Additional worker: 1

- Number Out: 204

- Total Cost: 34,841

- Idle Cost: 4,329

Step 3: Minimum of "Idle Cost"

This result shows that "Idle Cost" in "Total Cost" is 1/8. Additionally, compared with Case A,

- Number Out: 64 (increase)

- Total Cost: 678 (decrease)

- Idle Cost: 9,396 (decrease)

As a result, Case $B$ is better. Especially, it shows a great reduction in "Idle Cost". It can become even $1 / 3$.

Case $C$ : how many workers should be added and how long should they be employed as optimal simulation if additional members are 4 part-time workers? 
As the result, the value of 166 th simulation is the minimum. The result shows the following.

- Additional workers: 3 (1st worker: 2 hours, 2 nd: 2 hours, 3 rd: 4 hours)

- Total Cost: 35,344

- Idle Cost: 4,346

- Number Out: 205

There are some differences from "Total Cost" in compared with Case B. The result of Case B is better than that of Case $C$. In other words, the result shows that to add temporary workers who can work for 8 hours is better than to add part-time workers can work for 4 hours in this case.

\section{Conclusion}

Recently, in order to survive, companies cannot win the severe competition without doing reformation to adapt the change. The methodology proposed here fulfills the important role in executing BPR. This paper has focused on process simulation method and $\mathrm{ABC}$ analysis, and proposed a unified approach into which they are integrated. This paper has also presented a case study, in order to improve the effectiveness of the unified approach.

In present, we are collecting some successful example of BPR application and in the same time, doing some research to develop new methodologies, which apply information technology to BPR. This has been done, as we want to consider how to mix information technology with BPR to measure the efficiency of the organization after changing the organization itself or its way of work.

\section{References}

1. Hammer, M. and J. Champy., Reengineering the Corporation: A Manifesto for Business Revolution, (Harper Collins Publisher, Inc, New York, 1993).

2. Chen, M., BPR Methodologies: Methods and Tools, in D.J. Elzinga, et al. eds, Business Process Reengineering, Kluwer Academic Publisher, 187-211 (1999).

3. Pidd, M., Tools for Thinking: Modeling in Management Science, (John Wiley \& Sons, Inc, New York, 1999).

4. Brimson, J.A., Activity Accounting: An Activity-Based Costing Approach, (John Wiley \& Sons, Inc, New York, 1991).

5. M. Ozbayrak, M. Akgun, and A. K. Turker., Activity-Based Cost Estimation in a Push/Pull Advanced Manufacturing System, International Journal of Production economics, 87,49-65 (2004). 